\title{
Wavefront sensing detectors: 2 decades of technological progress
}

\author{
Philippe Feautrier ${ }^{1,2}$, Jean-Luc Gach ${ }^{1,3}$, Eric Stadler ${ }^{1,2}$, Fabien Clop ${ }^{1}$, Stephane Lemarchand ${ }^{1}$, \\ Thomas Carmignani ${ }^{1}$, Yann Wanwanscappel ${ }^{1}$, Xavier Girard ${ }^{1}$, David Boutolleau ${ }^{1}$ \\ ${ }^{1}$ Univ. Grenoble Alpes, IPAG, F-38000 Grenoble, France; \\ ${ }^{2}$ First Light Imaging SAS, 100 route des Houillères, 13590 Meyreuil, France; \\ ${ }^{3}$ LAM, Laboratoire d'Astrophysique de Marseille, Technopôle de Château-Gombert - 38, rue \\ Frédéric Joliot-Curie -13388 Marseille, France;
}

\begin{abstract}
New disruptive technologies are now emerging for detectors dedicated to adaptive optics. The detectors needed for this kind of applications need antonymic characteristics: the detector noise must be very low but at the same time must also sample the fast temporal characteristics of the signal. This paper describes the new fast low noise technologies that have been recently developed for adaptive optics.

The first technology is the Avalanche PhotoDiode (APD) infrared arrays made of HgCdTe. In this paper are presented the two programs that have been developed in that field: the Selex Saphira 320x256 [1] and the 320x255 RAPID detectors developed by Sofradir/CEA LETI in France [2],[3], [4]. Status of these two programs and future developments are presented. Sub-electron noise can now be achieved in the infrared using the e-APD technology. The exceptional characteristics of $\mathrm{HgCdTe}$ APDs are due to a nearly exclusive impaction ionization of the electrons, and this is why these devices have been called "electrons avalanche photodiodes" or e-APDs. These characteristics have inspired a large effort in developing focal plan arrays using HgCdTe APDs for low photon number applications such as active imaging in gated mode (2D) and/or with direct time of flight detection (3D imaging) and, more recently, passive imaging for infrared wave front correction and fringe tracking in astronomical observations. A commercial camera solution called C-RED One, based on Selex Saphira and commercialized by First Light Imaging [5], is presented here. In addition, First Light Imaging also developed the C-RED 2 InGaAs 640x512 400 fps infrared camera for lower budgets.

In the visible, another disruptive technology is showing outstanding performances: the Electron Multiplying CCDs (EMCCD) developed mainly by e2v technologies in UK. The OCAM2 camera, commercialized by First Light Imaging [5], uses the 240x240 EMMCD from e2v and is now routinely and successfully implemented on several world class telescopes.
\end{abstract}

Keywords: Adaptive optics, EMCCD, Avalanche photodiodes, APD, HgCdTe, SAPHIRA, SELEX, wavefront sensor, infrared sub-electron noise.

\section{INTRODUCTION}

Developed by First Light Imaging and based on the Saphira detector developed by Selex for ESO, the C-RED infrared camera is opening a new era in terms of sensitivity and speed in the SWIR scientific cameras domain and is particularly suited for infrared wavefront sensing in complex AO systems like MCAO. This is in strong contrast to what is observed in APDs made out of III-V material or Si, which requires high inverse bias and have typical noise factors of $\mathrm{F} \sim 4-5$ for IIIV semi-conductors and F 2-3 for Si respectively. These exceptional characteristics of HgCdTe APDs are due to a nearly exclusive impaction ionization of the electrons, why these devices have been called electrons avalanche photodiodes, eAPDs [6]. The next section presents the two main programs (RAPID and SAPHIRA) that have been developed around this new disruptive technology. 


\section{THE RAPID E-APD INFRARED WAVEFRONT SENSING DETECTOR}

\subsection{Presentation of the RAPID research program}

Developed by the SOFRADIR and CEA/LETI manufacturers, the RAPID infrared detector offers a $320 \times 2558$ outputs 30 microns e-APD array, sensitive from 0.4 to 3.3 microns, with less than 2 e readout noise at 1600 fps. Advanced packaging with miniature cryostat using pulse tube cryocoolers was developed in the frame of this programme in order to allow use on this detector in any type of environment. In 2013, the partners delivered the first prototypes and, given the performance results of these prototypes, the decision was quickly taken to push for an on-sky demonstration on a demanding instrument. PIONIER was chosen as its interferometric combination of light requires a very fast detector to fight against atmospheric turbulence, and a minimum amount of noise in order to detect faint objects. The RAPID detector is now implemented on the PIONIER [7] instrument on the ESO/VLTI interferometer in Paranal since December 2014. Since this time, RAPID observed more than 150 stars during more than 45 nights on the VLTI with a tremendous gain compared the previous camera based on conventional MCT IR detectors.

\subsection{The RAPID 320x255 pixel e-APD array presentation}

The RAPID programme was a 4 years R\&D project funded by the French "Fonds Unique Interministériel" in 2009. It includes several industrial and academic partners from the field of advanced infrared focal plane arrays fabrication (SOFRADIR, CEA-LETI) and of astronomical/defense institutes (IPAG, LAM, ONERA). The goal of this programme is to develop a fast and low noise infrared focal plane array of moderate format for astronomical fast applications. This research programme is currently ongoing with FOCUS funding.

The main characteristics of RAPID are:

- $\quad$ Pixels Format: 320 x 255 pixels $30 \mu \mathrm{m}$ pitch

- $\quad$ Technology: HgCdTe, intra-pixel CDS and CTIA, sensitive from the visible to 3- $3.3 \mu \mathrm{m} @ 77 \mathrm{~K}$

- $\quad$ Rectangular window can be defined with the start line and the end line of the window to be read.

- $\quad$ Noise: less than 2 e.

- $\quad$ Frame rate: $1500 \mathrm{~Hz}$, up to $2000 \mathrm{~Hz}$

- $\quad$ Dark signal: 100 e-/s measured, limited by setup background

- $\quad$ Power consumption: $122 \mathrm{~mW}$

The goal of the RAPID development was to demonstrate operation of the 320x255 pixels 30 microns pitch infrared array at 2000 fps with less than 2 e- readout noise. To achieve such readout noise and fast frame rate, APDs technology and intra-pixel Correlated Double Sampling were both needed. The floor plan of the device is shown in the Fig. 1, it includes 8 parallel outputs clocked at $20 \mathrm{MHz}$ pixel rate defining 8 stripes of $40 \times 256$ pixels with one amplifier per stripe. The detector can be seen in the Fig. 2 during its integration in the pulse tube cryostat. 


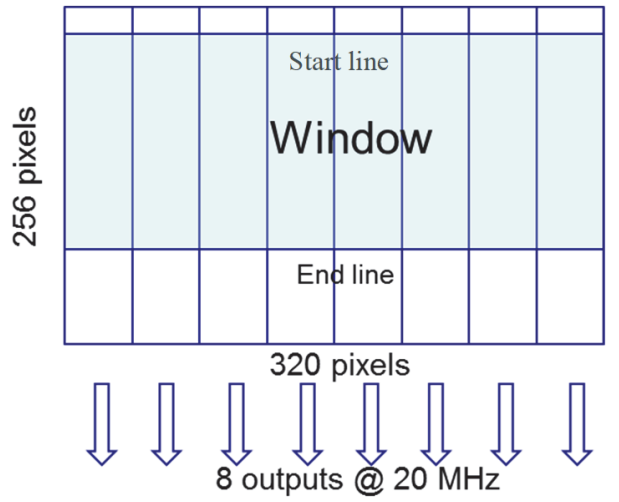

Fig. 1: The 1.6 kfps RAPID e-APD infrared detector configuration: 8 outputs $320 \times 255$ pixels with $30 \mu \mathrm{m}$ pitch. A rectangular window with programmable start line and end line can be defined to speed up the frame rate.

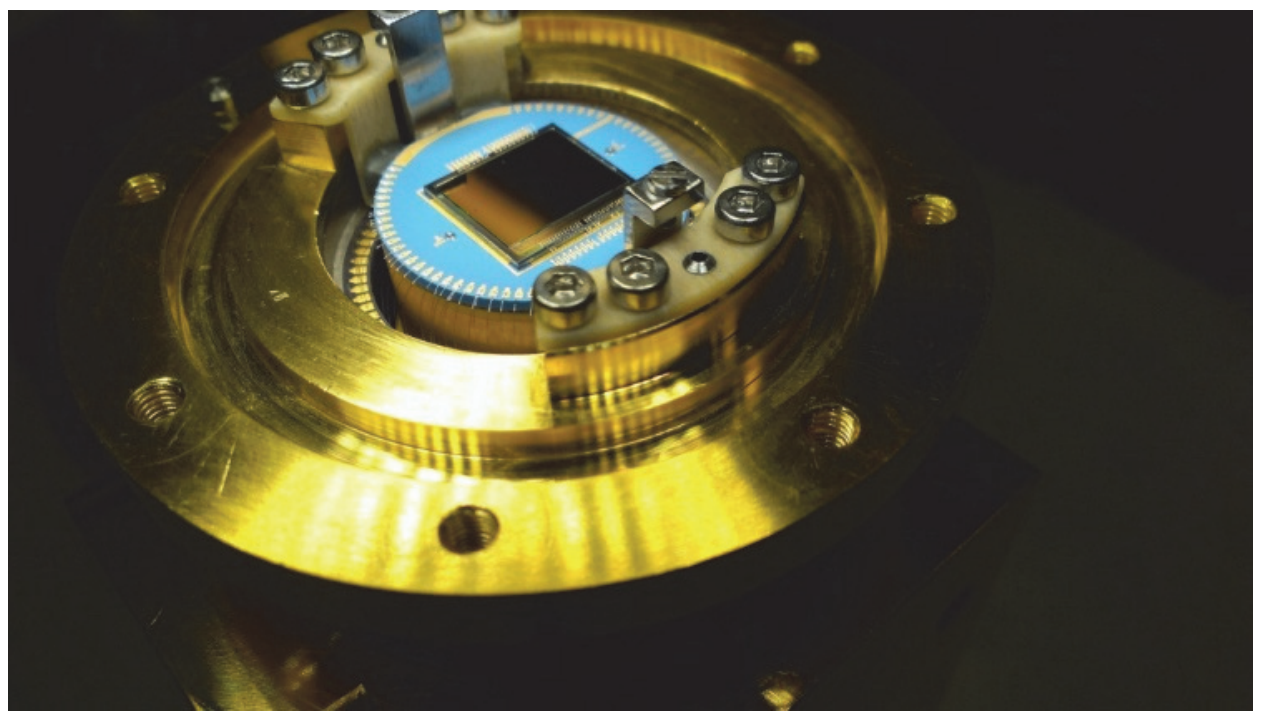

Fig. 2: The RAPID 320x255 IR APD array during integration by Sofradir in its cryostat cooled with a miniature pulse tube developed by the RAPID programme.

\subsection{RAPID detector results}

The best performances compromise for RAPID was to use $3.3 \mu \mathrm{m}$ cutoff photodiodes providing multiplication gain exceeding 25 and providing readout noise below 2 e. The current readout circuit of the detector limits the photodiode polarization to $8 \mathrm{~V}$. 


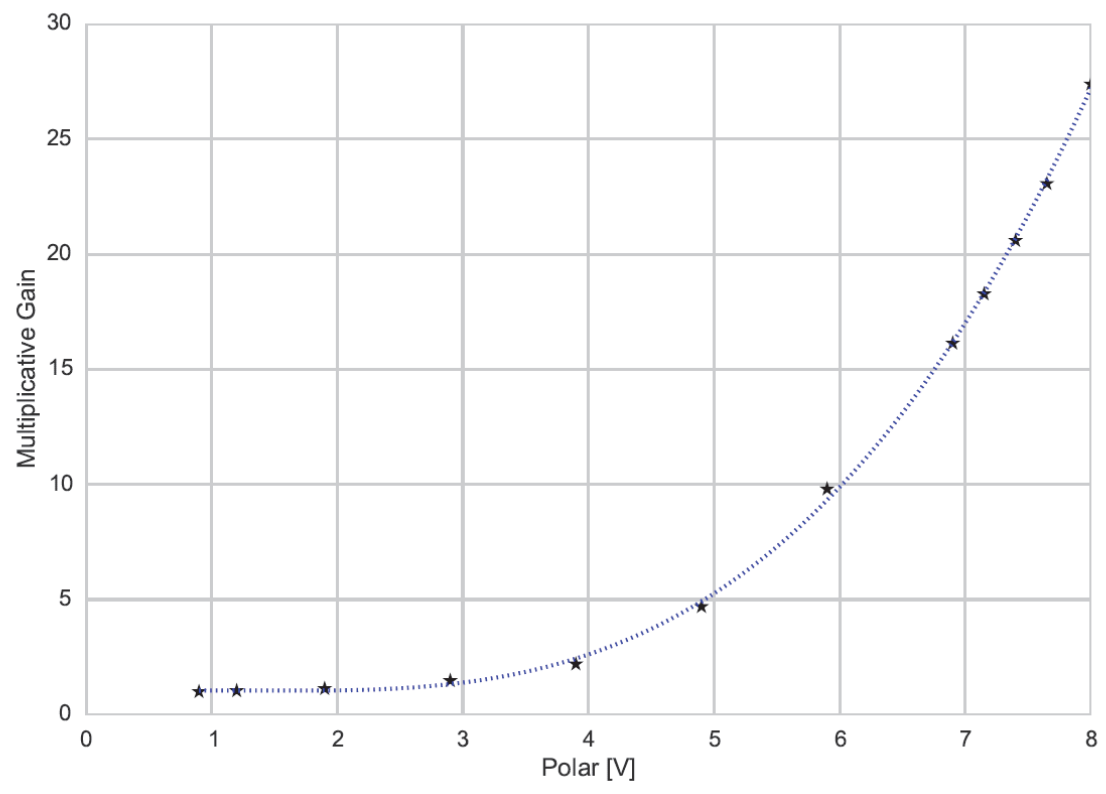

Fig. 3: mean multiplication gain of the APD array as a function of the photodiode polarization voltage for $3.3 \mu \mathrm{m}$ photodiodes cut-off.

The Fig. 3 shows the multiplication gain as function of the photodiode voltage for the RAPID $3.3 \mu$ m cut-off array. As the cut-off wavelength is large, this allows to obtain high multiplication gain for a modest photodiode polarization. Sub-e readout noise for the maximum photodiode polarization can then be measured as it can be seen in the Fig. 4 .

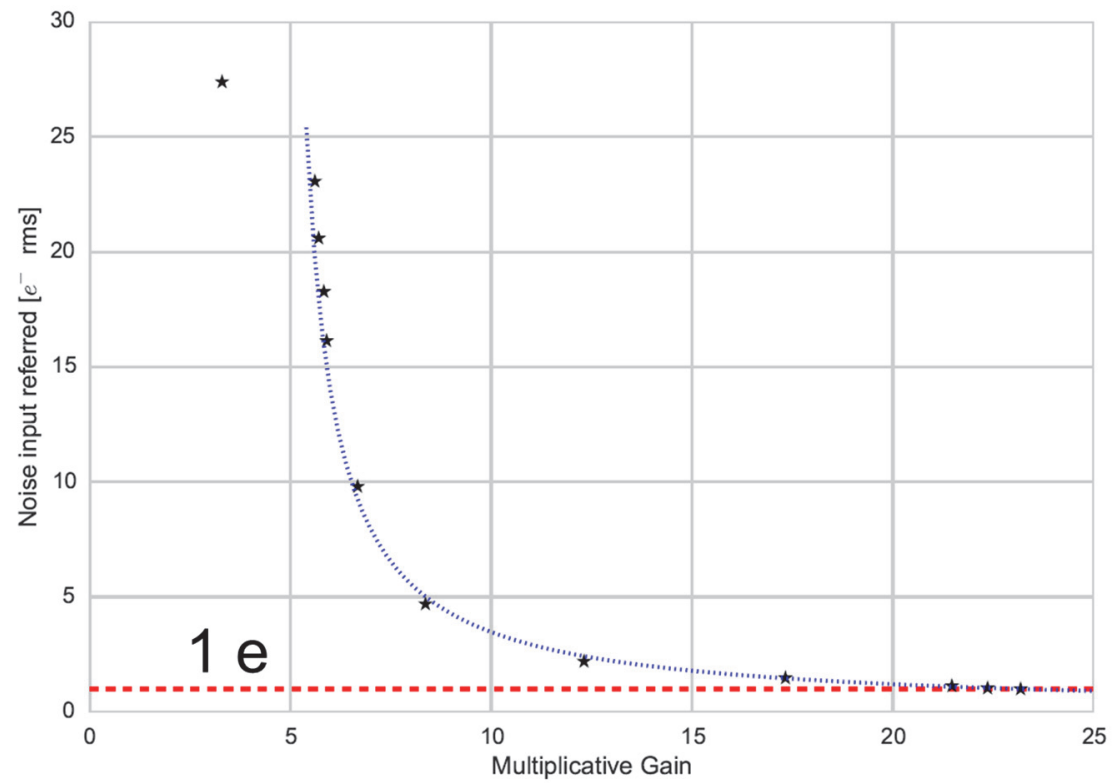

Fig. 4: mean readout noise as a function of the multiplication for RAPID array with $3.3 \mu \mathrm{m}$ cut-off. 


\section{THE C-RED ONE INFRARED APD CAMERA FROM FIRST LIGHT IMAGING}

\subsection{The C-RED One infrared APD camera presentation}

C-RED One is the only one commercial infrared camera using the Selex Saphira $320 \times 256$ pixels HgCdTe e-APD array with 24 microns pixel pitch. C-RED One is developed by First Light Imaging [5]. The sensor cutoff wavelength is 2.5 microns and it allows sub-electron readout noise, taking advantage of the e-APD noise-free multiplication gain and non destructive readout ability. C-RED One is also capable of multiple regions of interest (ROI) readout allowing faster image rate (10's of $\mathrm{KHz}$ ) while maintaining unprecedented sub-electron readout noise.

The sensor is placed in a sealed vacuum environment and cooled down to cryogenic temperature (70K) using an integrated pulse tube, with a high reliability (MTBF $>90000 \mathrm{~h}$ ) much higher than standard Stirling coolers used usually with cooled infrared arrays.

The Fig. 5 shows a picture of the C-RED One camera. On top of the camera, the helium compressor for cooling the pulse tube can be seen on this figure.

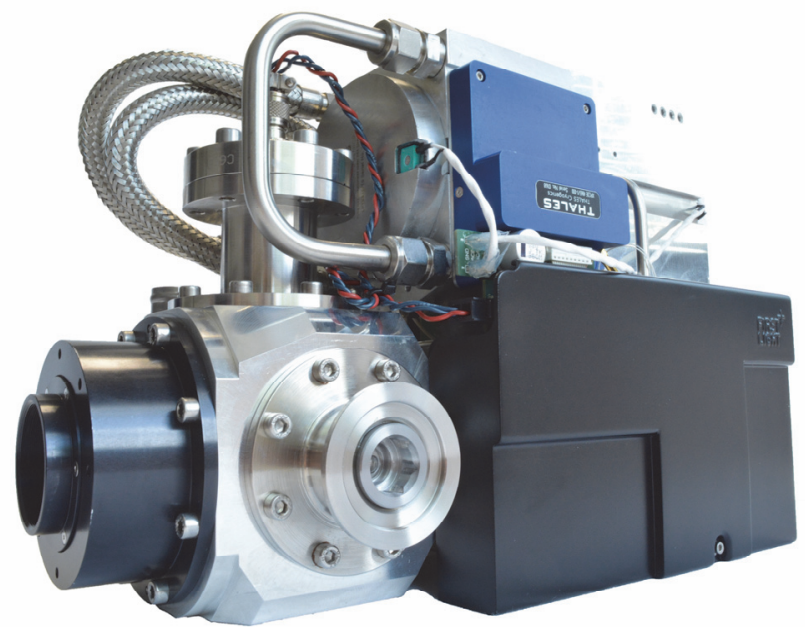

Fig. 5: the C-RED One infrared camera commercialized by First Light Imaging and that uses the Selex Saphira infrared array.

\subsection{The Selex Saphira detector of C-RED One.}

Designed and fabricated by Leonardo (formerly Selex), the Saphira detector is designed for high speed infrared applications and is the result of a development programme alongside the European Southern Observatory on sensors for astronomical instruments. It delivers world leading photon sensitivity of $<1$ photon rms with Fowler sampling and high speed non-destructive readout ( $>10 \mathrm{~K}$ frame/s). Saphira is an $\mathrm{HgCdTe}$ avalanche photodiode (APD) array incorporating a full custom ROIC for applications in the 1 to $2.5 \mu \mathrm{m}$ range.

Like the RAPID detector, SAPHIRA uses the HgCdTe APD properties, offering sub-electron noise with multiplication gain up to $\mathrm{x} 80$. The pixel format is $320 \times 256$ pixels with $15 \mathrm{fF}$ integration node capacitance ( $30 \mathrm{fF}$ with $\mathrm{HgCdTe}$ diode). The array has 32 parallel video outputs, organized as 32 sequential pixels in row. The 32 outputs are arranged in such a way that the full multiplex advantage is available also for small sub-windows. Non-destructive readout schemes with subpixel sampling are possible. This reduces the readout noise at high APD gain well below the sub-electron level at frame rates of $1 \mathrm{KHz}$. The growth technology used now is the metal organic vapour phase epitaxy (MOVPE). This growth technology provides more flexibility for the design of diode structures. It is possible to make heterojunctions with different bandgap properties between the absorption region and the multiplication region. The change to MOVPE resulted in a dramatic improvement in the cosmetic quality with $99.97 \%$ operable pixels at an operating temperature of $85 \mathrm{~K}$. The avalanche gain is controlled by an external voltage. The digital and analog functions are controlled by a serial interface. The readout of 
Saphira allows to read multiple windows, each independently resettable. Glow protection and APD protection circuit are also included.

The Fig. 6 shows the functional bloc diagram of the ME1000 SAPHIRA readout circuit used currently in C-RED. ME1000 is the last version of the Saphira readout circuit. The ME1000 scanning modes include a Read-Reset-Read per row function, so the user can have complete control of the correlated-double-sampling process. Saphira ME1000 incorporates also glow suppression by using 100\% metal screening. A reset current limit function has been added in this readout circuit version to protect the array from short circuit APDs.

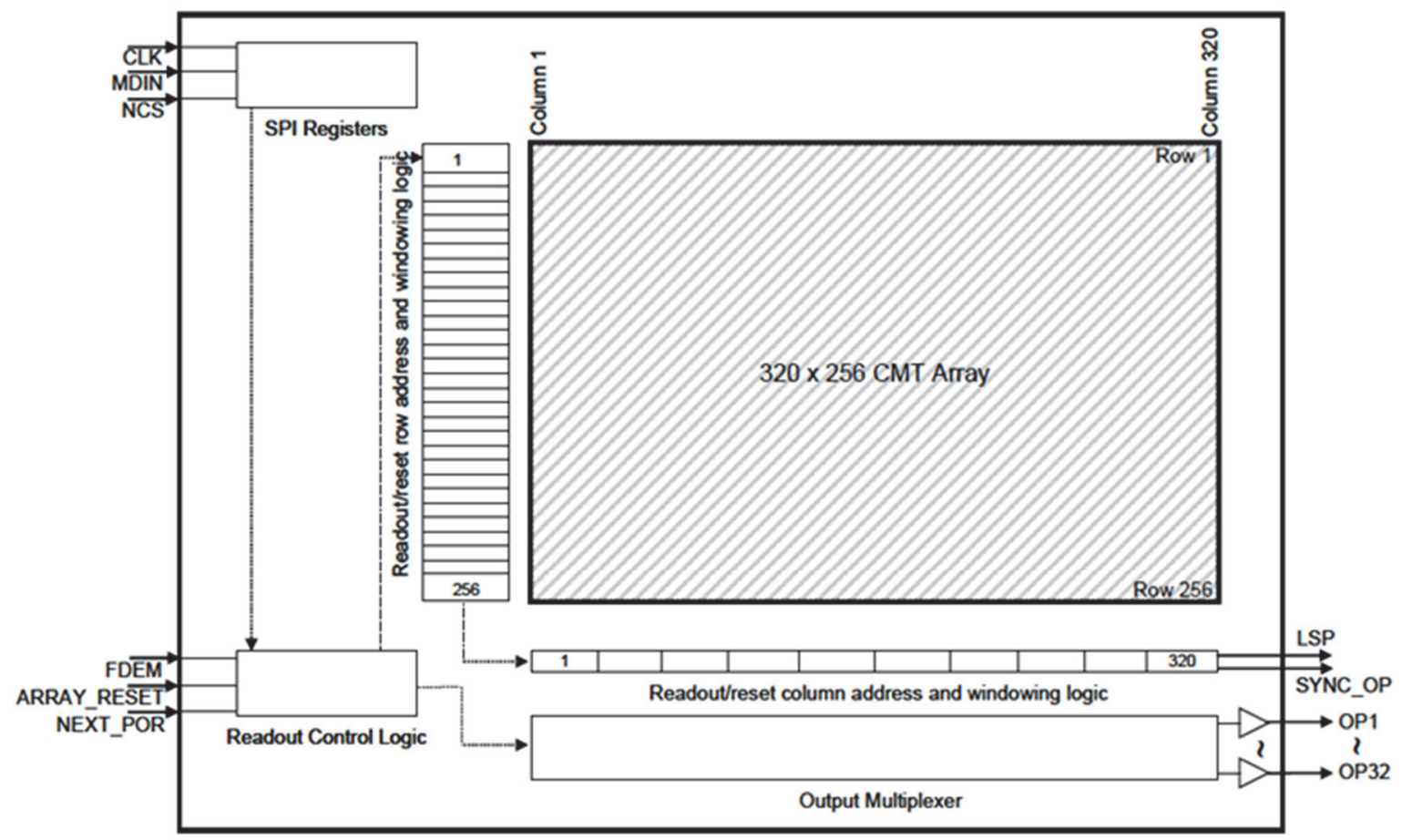

Fig. 6: the SELEX SAPHIRA ME1000 readout circuit architecture.

\subsection{The C-RED One camera characteristics and performances}

The C-RED one camera has the following main characteristics:

- MCT near IR Avalanche Photo Diode 320X256 with Selex Saphira detector.

- Sub-electron readout noise,

- 32 outputs, up to $3500 \mathrm{fps}$ in single read mode.

- Mean Readout noise at $3500 \mathrm{fps}$ and gain $60<1 \mathrm{e}$.

- $70 \% \mathrm{QE}$.

- Supported readout modes: read-reset-read per row, non destructive readout, rolling reset, CDS with read-resetread per row, multiple line/pixel read

- $\quad$ Pulse tube packaging cooling down to $70 \mathrm{~K}$

- Custom design available (beam aperture) 
- Cameralink full data interface

\subsection{Measured performances}

\section{Quantum efficiency}

The array quantum efficiency peaks up to near $70 \%$ and the array AR coating may be optimized on a per-device basis for J, H or K bands. Fig. 7 shows the effect of this QE optimization. Moreover due to junction heterostructure with $3.5 \mu \mathrm{m}$ cutoff wavelength $\mathrm{HgCdTe}$ material for the avalanche multiplication region and $2.5 \mu \mathrm{m}$ material for the absorber, the device is sensitive in L band at gain 1 but not with APD gain. This is due to photon penetration depth (longer wavelength photons penetrate deeper in the material and therefore are less amplified). We've measured that already with low gains (in the range of 5 to 10), the $\mathrm{L}$ band sensitivity is decreased to near zero, leaving only $\mathrm{J}, \mathrm{H}$ and $\mathrm{K}$ sensitivity.

\section{Quantum efficiency for Mark 13 APD arrays}

As a $\mathrm{f} n$ of antireflection coating thickness

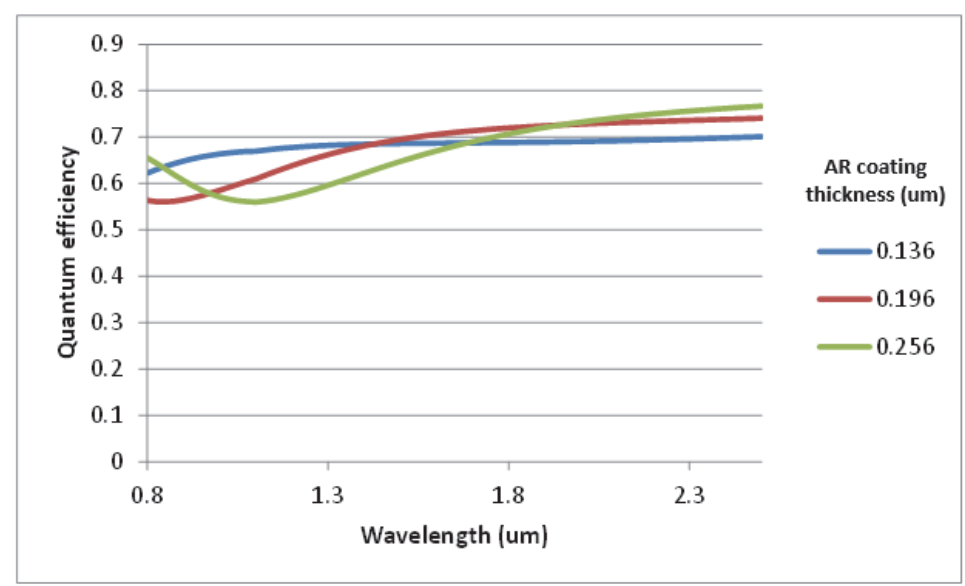

Fig. 7: AR coating and QE optimization for J, H or K bands of Mark13 e-APD diodes.

\subsection{System noise}

The noise measurement is done by measuring the temporal variation of the image, sensor in the dark looking at a Room temperature blackbody. Measurements have been done for single readout and multiple non-destructive readouts on x-axis and for gain from 1 to 300 on y-axis.

Taking into account $28 \mathrm{fF}$ node capacitance, the KTC noise should be in the range of 35e- at $80 \mathrm{~K}$. Fig. 8 shows the sensor readout noise as a function of the readout speed and multiplication gain. The readout speed variation is obtained by using either single read mode of multiple non destructive readout. It can be noticed also that for gains $>30$, the array enters in subelectron readout whatever is the readout mode (single readout or CDS). This is really a change of paradigm in the way of operating infrared arrays since CDS is no more needed to minimize readout noise, simply by increasing the APD gain, one can have very low noise operation, without compromise on readout speed, but at the expanse of a lower dynamic range. 


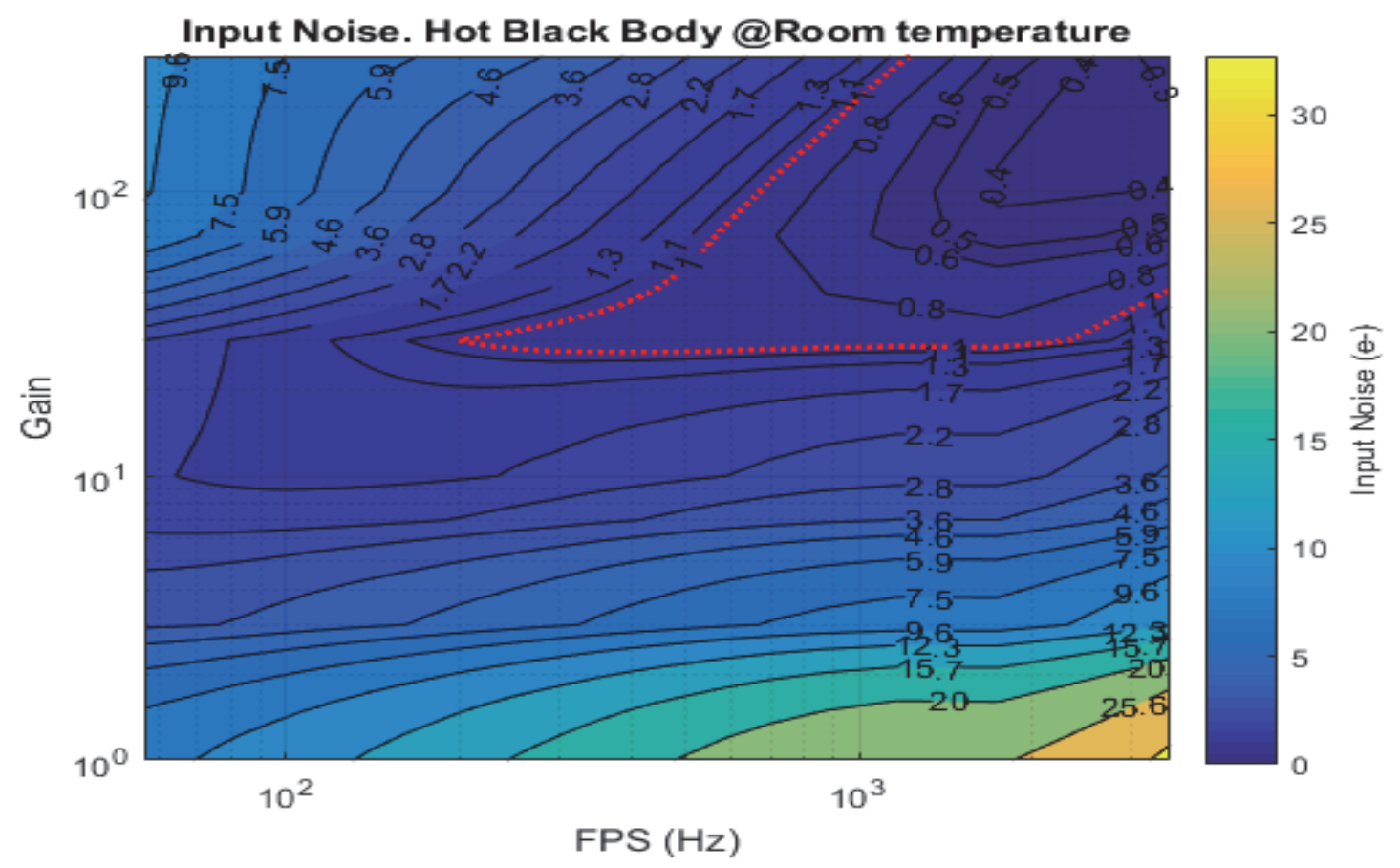

Fig. 8: Measured input referred readout noise of C-RED One vs APD gain and frame rate.

\section{THE C-RED 2 640X512 InGaAs SWIR camera from First Light Imaging}

C-RED 2 is a high performance, high speed low noise camera designed for Short Wave InfraRed imaging based on the SNAKE detector from Sofradir. Thanks to its state of the art electronics, software, and innovative mechanics, C-RED 2 is capable of unprecedented performances: up to 400 images per second with a read out noise from 10 to 30 electrons. To achieve these performances, C-RED 2 integrates a 640 × 512 InGaAs PIN Photodiode detector with $15 \mu \mathrm{m}$ pixel pitch for high resolution, which embeds an electronic shutter with integration pulses shorter than $10 \mu \mathrm{s}$. C-RED 2 is also capable of windowing and multiple regions of interest (ROI), allowing faster image rate while maintaining a very low noise.

The software allows real time applications, and the interface is CameraLink full and superspeed USB3. C-RED 2 is designed to be updated remotely, and needs no human assistance to manage the cooling. The camera can operate in very low-light conditions as well as remote locations. Designed for high-end SWIR applications, smart and compact (see Figure 1), C-RED 2 is operating from 0.9 to $1.7 \mu \mathrm{m}$ with a very good Quantum Efficiency over 70\%, offering new opportunities for industrial or scientific applications. 


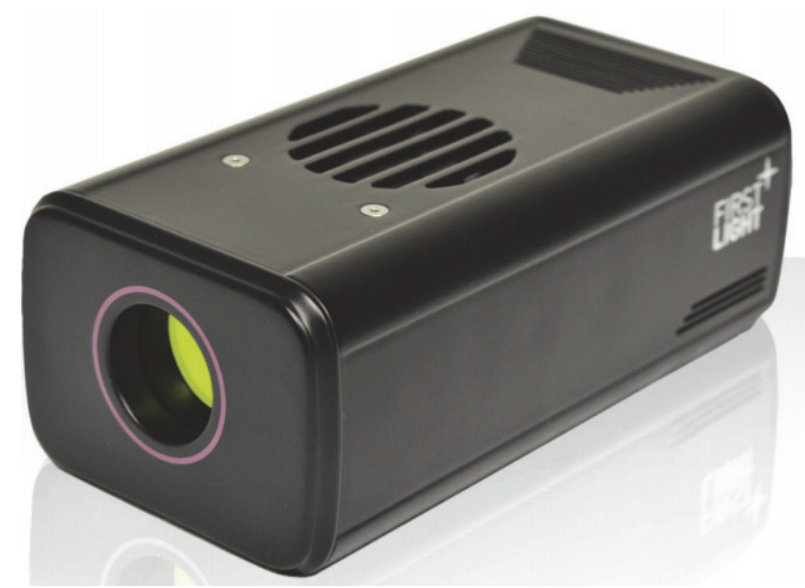

Figure 1: Picture of the C-RED2 camera

The Table 1 summarizes the main features and preliminary performances of the CRED-2 camera.

\section{C-RED 2 PERFORMANCES}

\begin{tabular}{|l|c|c|}
\hline Test measurement & Result & Unit \\
\hline Maximum speed & 400 & FPS \\
\hline Mean Dark + Readout Noise at 400 fps & 30 & e- \\
\hline Quantization & 14 & bit \\
\hline Detector Operating Temperature & -40 & $\mathrm{C}^{\circ}$ \\
\hline Flat Quantum Efficiency from 0.9 to $1.7 \mu \mathrm{m}$ & $>70$ & $\%$ \\
\hline Operability & 99.7 & $\%$ \\
\hline Image Full well capacity at low gain, 400 fps & 1400 & $\mathrm{ke}-$ \\
\hline Image Full well capacity at high gain, 400 fps & 43 & ke- \\
\hline
\end{tabular}

Table 1: typical performances and main features of the CRED-2 640x512 InGaAs SWIR camera.

\section{VISIBLE DETECTORS FOR ADAPTIVE OPTICS}

\subsection{The EMCCD technology and CCD220}

The CCD220 (see Fig. 9) is an L3Vision ${ }^{\mathrm{TM}}$ sensor fabricated by e2v technologies and designed for very high frame rate and low signal applications such as wavefront sensing or adaptive optics. The image area is split into two half sections for split frame transfer operation using metal-buttressed electrodes for high speed. The image section can be operated in inverted mode if desired. The device uses eight output amplifier circuits that are capable of operating at an equivalent output noise of less than one electron (rms) at frame rates of $>1.2 \mathrm{kHz}$. All outputs must be used for full image read-out. The e2v technologies back-thinning process ensures high quantum efficiency over a wide range of wavelengths, with a $>90 \%$ typical peak response. 
The device functions by converting photons to charge in the image area during the integration time period, then transferring this charge through one of the image and store sections into the readout register. Following transfer through the readout register, the charge is multiplied in the gain register prior to conversion to a voltage at the output amplifier. The multiplication gain may be varied by adjustment of the multiplication phase clock amplitude RØ2HV. The device is supplied in a sealed integral Peltier package, which provides a nominal operating chip temperature of $233 \mathrm{~K}\left(-40{ }^{\circ} \mathrm{C}\right)$. A variant of this device is also available with an integral shutter (CCD219).

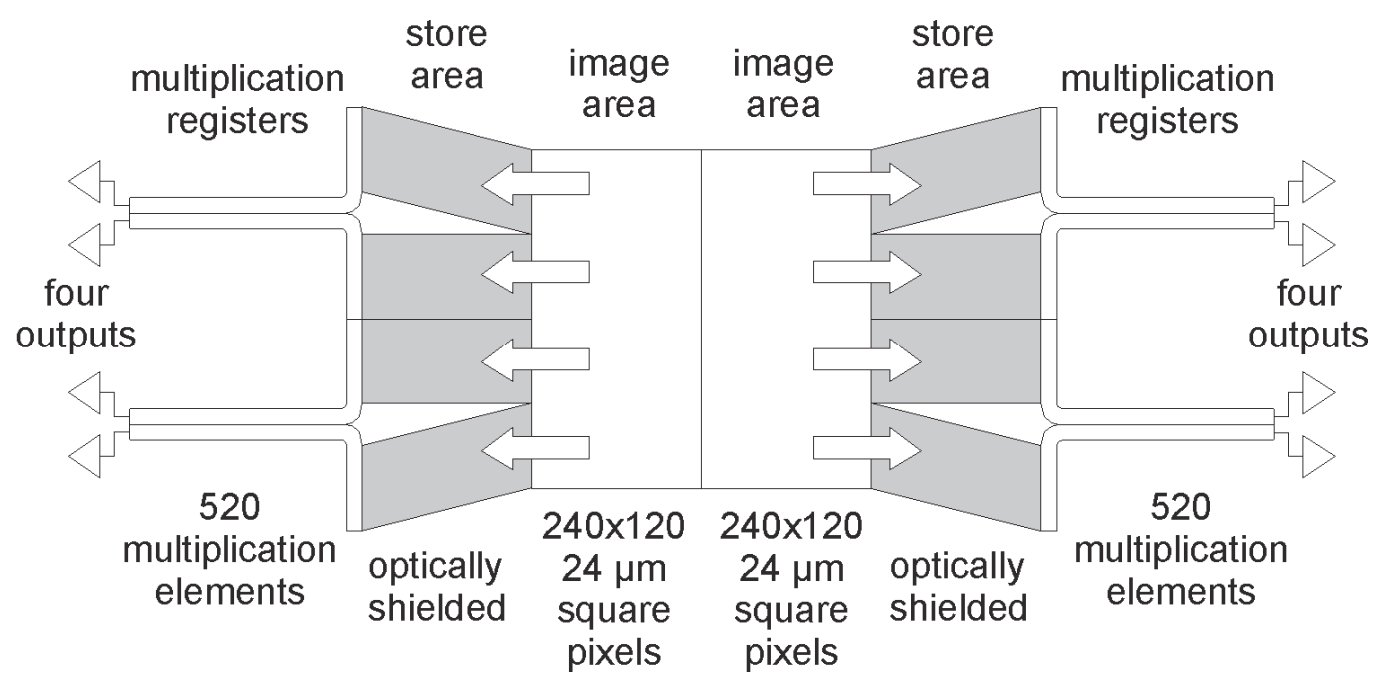

Fig. 9: Schematic of e2v technologies 240x240 pixel L3Vision CCD220. Eight Electron-Multiplying (gain) registers are used to obtain sub-electron noise at frame rates of $1300 \mathrm{fps}$.

\subsection{The OCAM2 camera based on the CCD219/220 EMCCD}

OCAM2, the production version of the OCam test camera, is commercialized by First Light Imaging. The OCAM2 system is capable of driving all members of the CCD220/219 family at their nominal speed (1.5kframes/s) and transmitting the data at full speed through a CameraLink interface. The camera controller is able to drive deep depleted variants with multilevel clocking at voltage levels up to $24 \mathrm{~V}$ with speeds of $10 \mathrm{Mlines} / \mathrm{s}$ (at a nominal phase load of $1 \mathrm{nF}$ ). To obtain such a speed, OCAM2 uses a special phase generation scheme. An arbitrary waveform generator is used. The core sequencer feeds a fast 14bit D/A converter running at $109 \mathrm{Mfps}$ followed by a class $\mathrm{AB}$ power amplifier that drives the CCD's phase. Using this generation method, it is possible to compensate for the parasitic PCB track/package pin inductance that makes a resonator with the CCD's phase and produce potentially destructive overshoots by using de-emphasis and suitable drive waveforms. This method can also be used to reduce the slew rate of the phase drive in order to minimize the generated Clock Induced Charges (CIC) [8].

The controller handles the $8 \mathrm{~L} 3$ vision outputs with high voltage clocking up to $50 \mathrm{~V}$ voltage swing. A big effort has been made to have high voltage stability (less that $1 \mathrm{mV} /$ hour of drift) in order to ensure a constant gain over a long period. The system digitizes the CCD signal using correlated double sampling with 14 bits resolution. Standard interfacing of the camera is performed by using a PC computer running Windows OS fitted with a CameraLink full grabber and a proprietary software capable of gathering in real time the extremely high data rate of $220 \mathrm{Mbytes} / \mathrm{s}$ produced by the camera.

\section{SIGNAL TO NOISE COMPARISON BETWEEN DETECTOR TECHNOLOGIES}

At various illumination levels, the Signal to Noise-Ratio (SNR) of various visible and infrared detectors is computed as: 


$$
S N R=\frac{S}{N}=\frac{Q E * S}{\sqrt{Q E * S * F+\sigma^{2}}}
$$

In this equation, the detector is assumed to be fast enough to have negligible dark signal. Here, $\mathrm{S}$ is the illumination signal (in photons/pixel/image), QE is the detector quantum efficiency, $\sigma$ is the readout noise, and $\mathrm{F}$ is the excess noise factor. A comparison of infrared detectors shows how much an e-APD sensor can improve the SNR for faint fluxes and also that its sensitivity in the infrared is directly comparable to EMCCDs in the visible - the latter of which are considered to be the most sensitive detectors (see Fig. 10).

Currently, the gain in performance of EMCCDs compared to classical CCDs in the visible is smaller than the gap between C-RED One and its competitors - whether they be slow-scan HgCdTe or even indium-gallium-arsenide (InGaAs)-based cameras. This advance in performance characteristics should allow e-APD imagers to usher in a new era in highperformance infrared detection.

\section{Signal to noise comparison of various detectors}

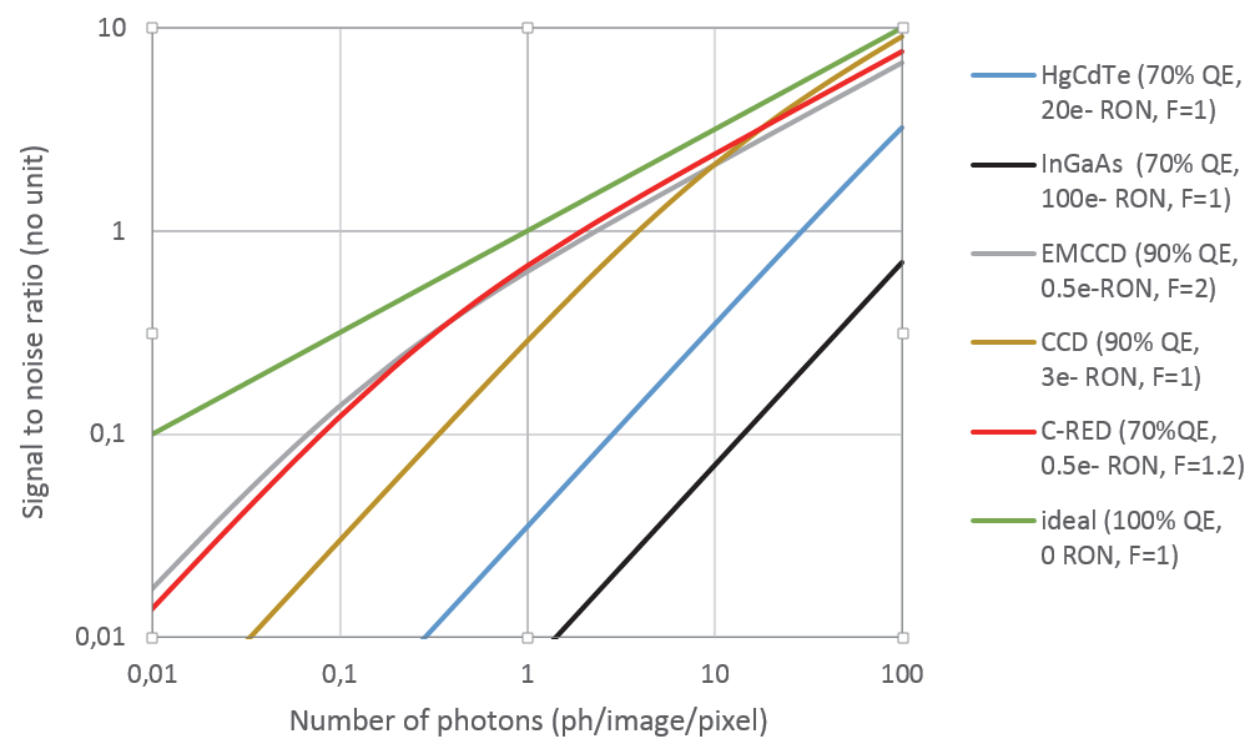

Fig. 10: Signal to Noise comparison of various detector technologies. CCD and EMCCD are sensitive in the visible. They are compared with IR detectors (InGaAs, slow scan HgCdTe and the C-RED e-APD camera).

\section{CONCLUSION}

In conclusion, the development of new detectors dedicated to fast applications is offering sub-electron readout in the visible and in the infrared, opening a new era in terms of sensitivity to the detectors for adaptive optics.

This paper illustrates a long term and coordinated fast detectors development in Europe involving cutting edge detectors and camera systems industry associated with ESO, academic French laboratories (LAM and IPAG) and the First Light Imaging spin off. Among these developments, a huge effort has been made on e-APD infrared arrays, together with the parallel development of state of the art camera systems using this disruptive technology.

One of these IR devices is called RAPID and is based on a $1.6 \mathrm{kfps} 320 \times 255$ pixels infrared APD arrays. This detector is now permanently installed on the ESO VLTI telescopes as focal plane camera for the PIONIER instrument. This is the first time a visible-infrared APD fast detector (1700 fps, 1 e noise ) is producing permanent astronomical data on a large world class telescope.

In the meantime, the C-RED 1 infrared camera is offered by First Light Imaging, taking advantage of the 320x256 Selex Saphira e-APD characteristics and performances in its latest and most advanced version. Sub-e noise at 3500 fps has been 
measured for the first time for an infrared device. This is the best compromise in terms of noise and speed for an infrared camera ever reported in the world.

In addition to the C-RED One development, C-RED 2 is InGaAs 640x512 fast camera with unprecedented performances in terms of noise, dark and readout speed based on the SNAKE SWIR detector from Sofradir. An incredible readout noise of $22 \mathrm{e}$ has been obtained at $400 \mathrm{FPS}$ readout speed in CDS mode. At $25 \mathrm{fps}$, the readout noise goes below $10 \mathrm{e}$. Cooled at $-40^{\circ} \mathrm{C}$, the C-RED 2 camera is able to achieve a dark current of $290 \mathrm{e} / \mathrm{s}(0.05 \mathrm{fA})$.

C-RED One and C-RED 2 are both SWIR commercial cameras from First Light Imaging fully available for ordering, in addition to the OCAM2 EMCCD based visible fast camera.

\section{ACKNOWLEDGMENTS}

The RAPID programme was funded by the Fonds Unique Interministeriel (FUI) under the 7th AAP from the French "Ministère de l'Economie, des Finances et de l'Emploi". Funding is also: ESO, CNRS, Université de Provence, Sofradir, ONERA and CEA/LETI. This work is also partially supported by the LabEx FOCUS ANR-11-LABX-0013. The authors thanks all the participants of the RAPID programme, among them are the industrial partners SOFRADIR and Biospace Labs, and the academic partners (IPAG, ONERA, LAM).

The infrared APD development at First Light Imaging has received funding from the European Union's Horizon 2020 research and innovation programme under grant agreement No 673944.

\section{REFERENCES}

[1] Gert Finger, Domingo Alvarez, Derek Ives, Leander Mehrgan, Manfred Meyer, Jörg Stegmeier, "SAPHIRA detector for infrared wavefront sensing", Proc. SPIE 9148, Adaptive Optics Systems IV, 914817 (August 7, 2014); doi:10.1117/12.2057078.

[2] P. Feautrier et al., "Advances in detector technologies for visible and infrared wavefront sensing", Proc. SPIE, 8447, 84470 Q (Sept. 13,2012).

[3] P. Feautrier et al., "Revolutionary visible and infrared sensor detectors for the most advanced astronomical AO systems", Proceedings of the SPIE, Volume 9148, id. 91481816 pp. (2014).

[4] J. Rothman et al., "HgCdTe APDs for space applications", ICSO 2014 - International Conference on Space Optics, 7 - 10 October 2014, Tenerife, Canary Islands, Spain.

[5] First Light Imaging SAS, http://www.firstlight.fr

[6] S. Derelle et al., "A Monte Carlo study of multiplication and noise in $\mathrm{HgCdTe}$ avalanche photodiodes," Proc. SPIE 7003, 70031P (Apr. 2008).

[7] LeBouquin, J.B.; et al.: "PIONIER: a 4-telescope visitor instrument at VLTI", 2011, A\&A, vol. 535, article id 67.

[8] "Low-light technical note 4 dark signal and clock-induced charge in 13vision ccd sensors," tech. rep., E2V Technologies, http://www.e2vtechnologies.com/secure/datasheets/13vision_ccds/low_light_tn4.pdf, June 2004. 